\title{
Crisis - what Crisis?
}

Persönliche Gedanken zur Corona-Krise aus der Leitungsverantwortung eines diakonischen Unternehmens

\section{Ambivalenz}

»In welcher Woche sind wir jetzt eigentlich? «, fragte ein Kollege in unserer täglichen Krisenstabssitzung. ${ }^{1}$ Corona ist längst zur Zeitenwende geworden, die in vor und nach teilt, die neue Begriffe wie ,Übersterblichkeit` und 'Lockdown` in die Alltagssprache getrieben und den Alltag selbst für uns alle sichtbar bis in unsere Gesichter hinein durch die partielle Maskenpflicht verändert hat. Wenn sich etwas in diesen Wochen als beständig erwiesen hat, dann ist es der Zwang, auf eine täglich sich ändernde Informations- und Erlasslage reagieren zu müssen. Wer immer über diesen Handlungsdruck hinaus meint, etwas fragend, kommentierend, deutend sagen zu wollen, sieht sich neben dem begründungsbedürftigen Ausbruch aus den gängigen Reaktionsmustern noch unter der Einschränkung, letztlich der Ambivalenz des Gesagten nicht entkommen zu können: »Nichts ist ohne sein Gegenteil wahr « (Walser 2012) - wie oft ist mir dieser Satz eingefallen angesichts sich widersprechender Expertenmeinungen, differierender Prognosen und notwendigem Komplexitätsaufbau.

Ambivalent und in einem strengen Sinn vorläufig bleibt auch alles persönlich Gedachte und Gesagte. Würde ich wie bisher denken und schreiben können, wenn ein mir nahestehender Mensch eine schwere Corona-Erkrankung erlebte, schließlich sterben würde?

Wer in der Krise denkt und schreibt, macht sich verletzlich, weil er schon morgen widerlegt sein kann. Letztlich ist er darin dem, der handelt, gleich, mit dem Unterschied allerdings, dass Handeln offensichtlich alternativlos ist, während das Denken sich erst als alternativlos erweisen muss. Diesen Beleg zu liefern, beanspruchen die folgenden Gedanken nicht. Sie unterliegen deshalb nicht nur dem allgemeinen Irrtumsvorbehalt, sondern stehen unter eingestandener Irrtumsanfälligkeit angesichts einer Krise mit vielen neuen Herausforderungen. Sie sind geschrieben aus der Perspektive eines in der Diakonie leitend tätigen Theologen und Ökonomen. Als Diakoniewissenschaftler am Institut für Diakoniemanagement bin ich zugleich an einer multiperspektivischen Sicht auf Phänomene interessiert. Anfänglich, in der tatsächlichen Quarantäne geschrieben, sind meine Überle-

1 Die Evangelische Stiftung Alsterdorf ist ein diakonisches Unternehmen mit ca. 6500 Mitarbeitenden und einem Jahresumsatz von ca. 300 Millionen Euro. Haupttätigkeitsfelder sind Eingliederungshilfe, Gesundheitsdienstleistungen, Bildung und anderes mehr. Seit den ersten Marzwochen trifft sich taglich virtuell ein interdisziplinärer Krisenstab. 
gungen das Ergebnis eines Austausches mit vielen Menschen. ${ }^{2}$ Für mich ausgesprochen anregend sind persönliche Gespräche mit skandinavischen Freunden, insbesondere mit Blick auf den >schwedischen Sonderweg ${ }^{3}{ }^{3}$

\section{Corona - was ist das für eine Krise?}

'Kriseく verweist sprachlich auf `Entscheidung`, könnte also in geschärfter Form übersetzt werden mit zzugespitzter Entscheidungssituation‘. Krisen entstehen, wenn Grenzen erreicht werden, die wir sonst mit normalen Mitteln beherrschen können. >Pandemier scheint die Reichweite der Situation anzugeben: eine Entscheidungssituation, die die ganze Bevölkerung angeht. Wir alle spüren, wie viel Richtiges in diesem Verständnis liegt. Regelungen, Verordnungen, Erlasse, die plötzlich alle Menschen betreffen und Gültigkeit haben. Gilt dies global, so differenziert sich dies doch zugleich nach Ländern und Staaten aus. So agieren wir nationalstaatlich und in Deutschland sogar föderalistisch begrenzt auf eine globale Herausforderung, weil die notwendige Herstellung globaler Handlungsfähigkeit bisher eine unerledigte Menschheitsaufgabe geblieben ist. Es ist symptomatisch, dass wir das 25. Jubiläum des Schengener Abkommens bei wieder geschlossenen Grenzen >feiern`. Geradezu skurril wird es, wenn die Grenzen von Bundesländern Einzelreisenden zu unüberwindbaren Hindernissen werden. Wir haben die Flüchtlingskrise nicht global bewältigen können und wir haben in der Bewältigung der Klimakrise lediglich Ansätze globaler Entscheidungen gesehen. Wie viele Krisen werden wir uns noch leisten können, bevor wir global oder zumindest verbindlich transnational entscheiden und handeln können? Für mich wie für viele andere ist die Klimafrage der Lackmustest der globalen Bewährung.

Freunde weisen mich darauf hin, dass die Corona-Krise durchaus die globale Handlungsfähigkeit erhöht hat. Pandemiebezogene Daten werden ausgetauscht, Forschungsschwerpunkte und -ergebnisse transnational ansatzweise transparent gemacht, Strategien zur Eindämmung der Ansteckung im Ländervergleich analysiert. Dies festzustellen, heißt allerdings zugleich, dies einzuschränken. Denn gleichermaßen sind Tendenzen festzustellen, die Suche nach Lösungswegen aus der Krise vor allem aus der Perspektive nationaler Interessen zu betreiben und in einen Überbietungswettbewerb mit gelegentlichen Abwertungsperspektiven zu tre-

2 Neben vielen Kolleg*innen in unserem Unternehmen danke ich besonders Prof. Dr. Dierk Starnitzke und Prof. Dr. Johannes Rüegg-Stürm.

3 Die Darstellung des schwedischen Sonderwegs in den deutschen Medien unterliegt m. E. mehreren Ausblendungen. In Deutschland ist so weniger bekannt, dass (1.) die stationären Altenheime einen extremen Transformierungsprozess mit großen, teilweise sprivateequity<-finanzierten Konzernen erlebt haben. Außerdem (2.) gibt es einige demographische Unterschiede gerade in der letzten Phase der Hochaltrigkeit, in deren Folge Heime (noch mehr) die Funktion von Hospizen haben. Interessant ist (3.) auch, dass die Regierungsberatung in Schweden/weniger durch Virologen als durch Epidemologen erfolgt. 
ten. Offener Forschungsaustausch und koordiniertes Vorgehen sind allenfalls in Ansätzen zu erkennen. Sie mögen in einer Wissenschaftsperspektive selbstverständlicher sein als im politischen Raum, der immer wieder reflexartig mit Perspektiveingrenzungen arbeitet.

Bevor man die Frage beantworten kann, was bei Corona zur Entscheidung steht, nein genauer, was wir durch Corona ausgelöst entschieden wissen wollen, muss man sich fragen, was das eigentlich für eine Krise ist. Manchmal hat man in der öffentlichen Debatte den Eindruck, Corona sei so etwas wie eine Naturkatastrophe. Bei Nacht und Nebel über uns gekommen, gleich einem Tsunami, macht sie uns Menschen zu unschuldigen Opfern. Die Unvermitteltheit der >Naturkatastropher scheinen noch Statistiken zu bestätigen, die feste Zyklen von Epi- und Pandemien aufzeigen. ${ }^{4}$ Der Zuschreibung der Krise als unausweichliches Fatum scheint es zu entsprechen, die Krise als äußeren Feind zu betrachten, gegen den man Waffenarsenale in Anschlag bringen muss, sodass kriegsrechtsähnliche Eingriffe geradezu zwingend wirken. Ich bin sehr froh, dass unsere politischen HandlungsträgerInnen hier überwiegend sehr verantwortlich darstellen, dass im Augenblick sein muss, was eigentlich nicht und auf Dauer auf keinen Fall sein darf. Ich frage mich aber, was wir an Verschiebungen hinsichtlich Rechtsstaatlichkeit und Demokratie gegenwärtig riskieren, wenn wir nicht, gerade auch sprachlich, unglaublich hellhörig und sensibel bleiben. Dass ein Land wie Schweden so stark auf individuelle Verantwortung und freiwillige Solidarität setzt, macht mich zusätzlich nachdenklich. Ich freue mich über jede im Wortsinne grundlegende Grundrechtsbesinnung wie etwa vonseiten unseres Bundestagspräsidenten Wolfgang Schäuble, der aufgrund seiner Zugehörigkeit zu einer Risikogruppe unverdächtig klarlegt, dass Grundrechte sich auf gleicher Ebene stehend gegenseitig einschränken (vgl. Birnbaum/Ismar 2020). Nur die Würde des Menschen hat den Status einer norma normans, an ihr sind alle Maßnahmen des Gesundheitsschutzes oder der Freiheitseinschränkung auszurichten. Was dies für unsere konkrete Praxis bedeutet, wird später zu reflektieren sein. Entscheidend ist aber, dass wir diesen Grundrechtsdiskurs führen müssen und uns an die Einschränkung der Grundrechte nicht gewöhnen dürfen. Es darf keine sneue Normalität ‘ der dauerhaften Außerkraftsetzung von Grundrechten geben. Der Begriff ist irreführend, weil er nicht einmal für den Zustand vor der Krise reklamiert werden darf, für die Zukunft aber geradezu zynisch sein könnte.

Wir erleben schon jetzt, wie Corona bezogen auf den Grund der Krise wie auch den nachfolgenden Umgang mit ihr im Sinne eigener Interessen und Positionen benutzt wird. Schon jetzt wird die Krise als Munitionslager für den Angriff auf Globalisierung und Kapitalismus verwendet, wie etwa die Berichterstattung der `taz` zeigt (vgl. Lee 2020). In Ungarn erleben wir, wie die Krise zur endgültigen Abschaffung der Demokratie missbraucht wird (vgl. ORF 2020). Ich bin sicher,

4 Die These von bestimmten epidemischen Zyklen ist schon 1938 aufgestellt worden (vgl. Jusatz 1938). 
wenn erst die Kosten dieser Krise transparenter werden, wird die Stunde der einfachen Antworten beginnen und der Populismus neues Futter erhalten. Wenn es so weit sein wird, werden gute Argumente und Positionen wichtig sein: Corona war keine Naturkatastrophe, sie war auch kein zyklisch berechenbares Fatum. Nicht Corona selbst, allenfalls die Ausbreitung war ein globales Problem. Auf den unterschiedlichsten Ebenen wirft Corona eine grundlegende Frage auf. Das gilt vielleicht schon mit dem ersten Indiz seiner Übertragung im Kontext eines Wildtiermarktes, dann aber vor allem mit den radikalen Veränderungen so vieler Alltagsroutinen. Wie wollen wir leben? Da diese Frage vielleicht zu groß ist, ließe sie sich herunterbrechen auf die Frage: Was wollen bzw. müssen wir in der Art unserer Lebensgestaltung grundsätzlich ändern? Diese Herausforderung stellt sich schon jetzt mit Blick auf Kulturtechniken. Videokonferenzen statt physischer Treffen, Gewöhnung an Flugverzicht und Homeoffice stehen nur für eine Reihe von Veränderungen, die sich etablieren. Die notwendig zu stellende Frage zielt über diese Neuerungen hinaus. Die Änderung unserer Lebensgestaltung steht so für uns alle spürbar zur Entscheidung an. Wäre das als Wesen der gegenwärtigen Krise zugleich auch ihre Chance?

Wir merken zurzeit, zu welch radikalen Veränderungen wir fähig sind und welche immensen Ressourcen wir mobilisieren können. Die Einführung digitaler Techniken bei uns in der Evangelischen Stiftung Alsterdorf, in der Verwaltung, der Therapie von psychisch Erkrankten wie auch in ersten Ansätzen des Homeschoolings, weist eine Veränderungsgeschwindigkeit auf, die man in unserer Branche kaum für möglich gehalten hat. Noch interessanter erscheinen mir organisationale Veränderungen: Um die kollektive Handlungsfähigkeit des Unternehmens zu gewährleisten, haben sich binnen weniger Wochen neue Beratungs- und Entscheidungsgremien gebildet. So ist etwa aus einer täglichen interdisziplinären Taskforce von neun Personen zwischenzeitlich eine wöchentliche Steuerungsgruppe geworden. Die Notwendigkeit, schnell und bereichsübergreifend kommunizieren zu müssen, etwa um auf Erkrankungen zu reagieren oder schlicht die Anschaffung von Masken und Hygienekleidung zu organisieren, hat zu einem wöchentlichen Meeting von Geschäftsführungen geführt, die sich sonst nur zweimal im Jahr getroffen haben. Was zuvor an Impulsen zur Entwicklung einer lateralen Organisation schwer in die Umsetzung zu bringen war, erhielt in der Krise eine Einsichtigkeit, die weit über den Krisenstatus hinausweist. Allerdings gilt auch hier gleichzeitig das Gegenteil: Der Zeitdruck und die Dringlichkeit haben manche Entscheidung und viele Umsetzungen hierarchischer werden lassen. Pandemiepläne und Quarantäneentscheidungen sind nicht Ergebnisse anspruchsvoller kollegialer Verständigungsprozesse.

Meine Befürchtung ist, dass das Potenzial dieser schnellen Veränderungen verspielt werden kann und die Wiederherstellung des Status quo ante, die Sehnsucht nach der >Normalität wieder ihre ganz eigene Logik entfaltet. Dahinter steht neben allen wirtschaftlichen Interessen ein allgemeines Phänomen von Krisen: Krisen wollen überwunden werden, gerne auch vergessen und verdrängt. Wenn 
etwa in einer Einrichtung wie der unseren Inklusion der Leitstern der Arbeit und spezifisch der Treiber der Fachkonzepte für die Arbeit mit Menschen mit geistiger Behinderung war, dann ist es kaum erträglich, wenn man unter Quarantänebestimmungen und Abstandsregeln täglich Widersprüche leben und aushalten muss.

Ein differenziertes Lernen, was es positiv zu lernen und weiterzuentwickeln gilt und was als notwendige Ausnahme möglichst schnell überwunden werden muss, ist hier keineswegs trivial. Bis sich nachhaltig Veränderungen etabliert haben, werden wir eine anstrengende Phase haben, in der wir Verunsicherung aushalten und das Fragen kultivieren müssen.

\section{Lernen wir das Richtige aus der Corona-Krise?}

Die Antwort auf diese Frage ist kein einfaches und optimistisches Ja. Ist es nach unserer allgemeinen Erfahrung nicht so, dass wir zunächst einmal dazu neigen, nicht neu zu lernen, sondern uns in dem bisher Gelernten selbst zu bestätigen? Aber umgekehrt kennen wir auch die Erfahrung: Neu erzwungene Situationen können auch zu neuen Wahrnehmungen führen. Mit einer gewissen Grundskepsis nehme ich wahr, dass viele diese andere Seite hoffnungsfroh überschätzen. `Nach Corona wird nichts mehr sein wie früher.< Diese häufig zu hörende These scheint mir daher nur wie die Parole, die es schnellstmöglich zu widerlegen gilt. Dabei gibt es dafür, dass Individuen wie Gesellschaften zu solchen Veränderungen in der Lage sind, gute Beispiele (für mich immer paradigmatisch: die Abschaffung der Familienfehde als `Gerechtigkeitsprinzip`, vgl. Weizsäcker 1991), aber ein Selbstläufer aufgrund vereinzelter Wahrnehmungen sind sie nie. Sie müssen zu Gestaltungsprinzipien werden, die gelernt, eingeführt und geschützt werden müssen.

Deshalb glaube ich auch zunächst nicht daran, dass das andere Erleben von Natur in diesen Tagen unseren Umgang mit natürlichen Ressourcen verändern wird oder die veränderte Tagesgestaltung unser zukünftiges Freizeitverhalten. Ja, wir können großartige Beispiele aufzählen, wie Solidarität und Kreativität zu neuen Lösungen geführt haben. Aber ich neige hier, und das nicht aus grundsätzlichem anthropologischem Pessimismus, eher der Position zu, dass wir alle verpassten Flüge nachholen werden und die kollektive, soziale Fastenzeit in schneller Gewichtszunahme des Bisherigen vergessen machen. Anders verhält es sich mit Veränderungen, die sich bereits vorher abgezeichnet haben und durch das Erleben in der Krisenzeit einen immensen Begründungsschub erhalten haben. Wurde etwa bei psychischen Erkrankungen schon lange über Möglichkeiten und Grenzen der Telemedizin nachgedacht und erste Versuche unternommen, so wurden die Begleitung und Behandlung von Patienten via digitaler Hilfsmittel im Lockdown alternativlos. Ähnliches gilt, auch in der Sozialwirtschaft, für Homeoffice und digitale Dienstbesprechungen und Konferenzen. Sie fanden schlicht statt, wurden durch Mitarbeitervertretungen meist unkompliziert genehmigt und funktionieren. Digitalisierungsverantwortliche in der Sozialwirtschaft sprechen von einem gefühlten 
Sprung von drei bis fünf Jahren. Es mag sein, dass wir auch hier wieder gegenläufige Bewegungen erleben. Mindestens kurzfristig kann sich auch eine Ermüdung hinsichtlich Homeoffice und digitalen Begegnungspräferenzen zeigen. Auf Dauer aber werden wir den Schub digitalisierter Kommunikation allenfalls leicht verzögern.

Daneben wird es vielleicht einige Bereiche geben, in denen der schon bisher vorhandene Veränderungsdruck so groß geworden sein wird, dass ein Wandel nicht mehr aufzuhalten ist. Ob die >Helden des Alltags` aus der Krisenzeit die Gehaltsgewinner des neuen Alltags werden? - Durchaus denkbar und wünschenswert. Aber auch Gesellschaften ziehen sich gerne auf >bewährte< Verhaltenslösungen zurück. Wenn dann erst einmal wieder die Legitimation staatlicher Intervention abgelaufen ist, kann es bei einem enger werdenden Verteilungskampf durchaus sein, dass man wieder zu den alten Lösungen greift, etwa dem Setzen auf Marktmechanismen oder dem Rotstift im Bereich des Sozialen.

Jenseits der wenigen Selbstläufer werden wir für nachhaltige Veränderungen (neue Formen der Zusammenarbeit und Dienstleistung) hart arbeiten müssen. Ansonsten läuft alles schnell wieder auf die Wiederherstellung des Bisherigen einschließlich gängiger Dauerkontroversen mit den bekannten Selbstbestätigungsmechanismen hinaus. So wird schon jetzt kontrovers diskutiert, ob die Krisenbewältigung eine Bestätigung der Hierarchie ist oder umgekehrt belegt hat, dass nur laterale Expertisestrukturen den komplexen Herausforderungen einer Krise gewachsen waren.

Wenn wir, gleichgültig ob auf persönlicher, organisationaler oder gesellschaftlicher Ebene, zu guten Lernergebnissen kommen wollen, werden wir entscheiden müssen, an welchen wenigen (!) Punkten wir uns für eine Veränderung dessen, was wir für notwendig halten, einsetzen:

- Global (und ich weiß, hier kann ich das Träumen nicht sein lassen): Wenn wir mit der gleichen Wucht und Umsetzungsstärke das Thema (ich sage es klassisch theologisch) >Bewahrung der Schöpfung umsetzen würden, wie weit könnten wir hier in kürzester Zeit kommen? Nicht in erster Linie die wirtschaftliche Konsolidierung, die uns fraglos heftig herausfordern wird, sondern allem voran die ökologische Nachhaltigkeit auf der Agenda als Störfaktor jeder Normalbetrieblichkeit hochzuhalten, wäre das ein denkbarer Weg? - Ich bin mir nicht sicher, ob wir damit als einzelne Organisationen nicht auch spunkten könnten, wenn wir dies, vielleicht etwas unerwartet, als Learning aus der Krise sehen.

- Europäisch: Die Grenzen sind zur Zeit dicht wie nie in den vergangenen 25 Jahren. Das mag als Ausnahmezustand seinen guten Grund haben. Aber diese Trennlinien dürfen nicht zu Grenzen der Handlungsfähigkeit und Solidarität werden. Europa nicht nur als Zusammenschluss ungleichzeitiger Wirtschaftssubjekte zu sehen, sondern als Werte- und Solidargemeinschaft glaubhaft zu leben, ist meines Erachtens Gebot der Stunde. Käme uns der erlebte Zerfall 
Europas nicht teurer zu stehen als die Zinsnachteile einer gesamteuropäischen Krisen- und Sanierungsstrategie? Speziell im Brüsseler Kreis, einem Zusammenschluss von 13 Caritas- und Diakonieunternehmen, haben wir die Erfahrung gemacht, wie sehr Europa ein gemeinsames Lern- und Gestaltungsfeld ist.

- Rechtsstaatlich: Der Rechtsstaat hat in der Krisensituation eine Bewährungsprobe bestanden. Er hatte, gerade weil ihm nicht der Missbrauch der Macht unterstellt werden konnte, die Legitimation zu schnellem Handeln. In seiner Verfasstheit als Rechtsstaat hat er den Schutz des Lebens (besonders verletzlicher Gruppen) über die Ökonomie gestellt, eine Entscheidung, die zu Lebzeiten zu erleben sich viele nicht einmal zu erträumen gewagt hätten. Die in der Krise so verführerische Prävalenz der Exekutive wurde teilweise in der Öffentlichkeit als sich verfestigende Tendenz befürchtet. Aber schon auf die wenigen Wochen gesehen lässt sich erkennen, dass die Organe der Gewaltenteilung zu keiner Zeit ausgehebelt wurden. Im Gegenteil erleben wir einen Streit um die Geltung der Wahrnehmung der Grundrechte, der in Wucht und Relevanz den Rechtsstaat durchaus stärken kann. Sehen wir zu, dass das so bleibt. Die Einschränkung von Grundrechten muss, wo immer sie im Konsens und nur auf Zeit begründbar sein mag, eine erlittene Ausnahme bleiben und darf nicht zu einem Gewöhnungszustand werden. Es darf keine sneue Normalität ' geben, in der die Selbstbestimmung und das Recht auf Teilhabe von Menschen mit Behinderung und die Würde von alten Menschen dauerhaft eingeschränkt ist.

- Sozialstaatlich: Daseinsvorsorge versus Marktsteuerung, das war bis vor Kurzem ein Gebiet für Spezialisten. Jetzt haben wir gelernt, dass diese Frage über Leben entscheiden kann. Haben wir aus der Krise nicht gelernt, dass es gesellschaftliche Bereiche gibt, die nur auf Kosten der Menschen der kurzfristigen Angebotssteuerung (was eben nichts mit sozialer Marktwirtschaft zu tun hat) unterworfen sein werden? Vielleicht werden wir die Kehre noch rechtzeitig realisieren können, nach der das Gesundheitssystem in seinen Auslastungskapazitäten immer nur am betriebswirtschaftlichen Optimum ausgesteuert wurde. In der Krise haben wir dann auch kritisch sehen müssen, dass die Aufmerksamkeitsskala des Sozialstaates immer noch mehr oder weniger große Ausblendungsbereiche hat. Wir denken aufgrund unserer Erfahrungen besonders an die Eingliederungshilfe und die Hilfe für Wohnungslose. ${ }^{5}$ Glücklicherweise wurde diese Wahrnehmungsschwäche zunehmend korrigiert. Die betroffenen Gruppen hatten in uns (der freien Wohlfahrtspflege) ihre verlässlichsten Partner und auch Sozialanwälte. Können und müssen wir hier nicht mit ganz

5 Es gehörte zu den erschreckenden Erfahrungen der ersten Krisenwochen mitzuerleben, wie die Erfordernisse des >Distancing für Wohnungslose kaum bedacht wurden. Als Wohnungslosentreffs schlossen, gab es eben für diese Menschen nicht die Möglichkeit, sich in die eigene Wohnung zurückzuziehen. Eine andere Erfahrung war es, wie schwer die Notwendigkeit von Schutzkleidung für Mitarbeitende in der Eingliederungshilfe in der Anfangszeit zutvermittelin war. 
anderer Wucht als bisher das Modell des gemeinwohlorientierten Wirtschaftens als das Erfolgsmodell öffentlich vertreten?

- Unternehmerisch: Das Lernen steht hier noch ganz in den Anfängen, war der Handlungsdruck doch zu groß, gerade auch bei unseren sogenannten Komplexeinrichtungen, die Dienstleistungen in mehreren sozialen Feldern anbieten. Meine Meinung wage ich deshalb nur in Konjunktiv und Frage auszudrücken: Waren wir nicht da besonders erfolgreich für die Menschen, wo wir, etwa bei der Beschaffung von Schutzkleidung oder in der Versorgung von Covid-19-Klienten mit Behinderung, unser Säulendenken aufgegeben haben? Hat es sich so nicht als förderlich erwiesen, wenn man unter einem Dach beispielsweise Krankenhäuser und Einrichtungen der Eingliederungshilfe hat? Im Prinzip könnte sich auch die Verbindung von Arbeitsschwerpunkt vor Ort und Bündelungskompetenz in einer Zentrale bewährt haben. Kein Teil hat sich hier als entbehrlich erwiesen. Zugleich war aber auch erkennbar, dass die Entscheidungsstrukturen etwas breiter, flexibler und situativer aufgebaut werden können.

- Individuell: Meine Gedanken und Fragen meine ich hier nur als Einladung zur Selbstreflexion. Ich habe wieder einige Unterscheidungen liebgewonnen: Die Unterscheidung von Angst und Sorge. Angst hat kein klares Gegenüber, entgrenzt sich als lähmendes Lebensgefühl und beherrscht. Sorge hat ein klares Gegenüber, führt in Verantwortung und Handeln. Nicht immer kann ich diese Angst in Sorge überführen. Ich möchte aus der Krise lernen, Angst, wo immer dies möglich ist, in Sorge zu überführen. Für mich als Theologen verbindet sich dies mit dem Wissen um meine Bedürftigkeit: Ich brauche andere, letztlich im Glauben Gott selbst. Vermutlich werden wir den vor uns liegenden Phasen der >Normalisierung a anmerken, wie entscheidend die Überwindung der Angst ist. Nicht einmal das sogenannte Hochfahren der Wirtschaft wird gelingen, solange diese das beherrschende Lebensgefühl ist.

\section{Welchen Geist atmet unsere Krisenbewältigung?}

Wenn ich diese Frage gesondert herausstelle, klingt dies vielleicht sonderbar, in jedem Fall begründungsbedürftig. In der Argumentation werde ich auf einen theologischen Gedanken zurückgreifen, der mir in seiner Relevanz in den vergangenen Wochen immer stärker bewusst geworden ist.

Aber zunächst einmal etwas allgemeiner einsetzend: Jede große Menschheitskrise wurde mit bestimmten Deutungsmustern und mentalen Bearbeitungsweisen und unter Favorisierung von bestimmten Handlungstypen angegangen. Die mittelalterliche Pest wurde als göttliche Strafe für menschliche Schuld gedeutet, führte in eine Bußhaltung und favorisierte den Arzt und den Bußprediger. In einer pluralen und säkularen Gesellschaft mögen die Deutungen vielfältiger (deshalb aber nicht unbedingt richtiger) sein, aber die Grundmentalität ist die der aktiven 
Bekämpfung eines äußeren Feindes mit dem Primat des Krisenmanagers. Wie viel daran richtig und lösungsorientiert ist, muss nicht unterstrichen werden. Aber mindestens manchmal kommen mir Fragen: Die jetzige Krise zeigt die unglaubliche Verletzlichkeit nicht nur unseres Systems, sondern auch unserer menschlichen Existenz. Sie führt uns nicht zuletzt auch an Ohnmachtserfahrungen. Regen wir uns nicht deshalb so über Corona auf, weil wir mit unserer eigenen Begrenztheit konfrontiert werden? Nicht einmal die doch fast mathematisch zu erreichende Wahrscheinlichkeit der notwendigen Ressourcen zur Todesvermeidung haben wir weltweit im Griff. Die Pandemie treibt uns an die Grenze der Machbarkeit. Sterblichkeit und Endlichkeit sind grundsätzlich konzediert, aber der Übersterblichkeit können wir identitätsstiftend den Kampf ansagen. Wir erheben uns als die, die handeln müssen und können, die wissen, was zu tun ist, und sei dies auch nur in der Fristigkeit kürzester Zeiträume. In der Zerschlagung unserer gewohnten Handlungsmöglichkeiten erweisen wir uns als die entscheidenden HandlungsträgerInnen.

Dafür, dass die Handlung uns in unserer Identität trägt, kennt die Theologie den Begriff der Werkgerechtigkeit. Werkgerechtigkeit meint dabei klassisch, dass der Mensch sich durch sein eigenes Handeln vor Gott als gerecht und gut erweisen will. Dieses widerständige Sperrgut der Theologie eines Paulus und eines Luthers lebt davon, dass es nicht moralisch missverstanden wird. Das Problem des Handelns aus Werkgerechtigkeit besteht nicht darin, dass es moralisch falsch oder situativ unangemessen wäre. Das gute Werk bleibt gut, sowohl moralisch gesehen wie in Blick auf den, dem es zugute kommt. Es kann sogar alternativlos sein und somit geboten. Sein Problem besteht einzig darin, dass es identitätsstiftend genutzt wird. Statt sich etwa aus Passivität und Empfangen heraus zu verstehen, setzt die Werkgerechtigkeit auf die Selbstbestätigung durch Handeln. Durch das gute Werk gerecht vor Gott dazustehen, mag das Problem des mittelalterlichen Menschen gewesen sein. Der selbsterteilte Freispruch vor sich selbst und anderen durch das Handeln hat sich demgegenüber gehalten. Er funktioniert dort besonders, wo er >kartellbildend ‘ wird, wo die Bestätigung auf Gegenseitigkeit prägend wird.

Luthers radikale Sicht war hier die unglaubliche Zumutung, dass dieses moralisch gesehen fraglos gute Werk Sünde sei, also Ausdruck eines falschen Gottes-, Selbst- und Mitmenschenverhältnisses. In gleicher Entschiedenheit setzt er dagegen ein Handeln, das nur den Nächsten um seiner selbst willen im Auge hat.

Die Werkgerechtigkeit ist kein Handlungskonzept und kein Rezept zur Analyse von menschlichem Handeln. Für mich ist sie vor allem ein Entdeckungszusammenhang, der ohne zwingende Bindung an die theologischen Überzeugungsinhalte uns heilsam ablenkt von falschen Fixierungen. Ob er im Zusammenhang mit unserer Krise gesehen eine Erschließungskraft hat, mag jeder selbst für sich entscheiden. Was ich mir jedenfalls in dieser Krise erhoffe, möchte ich einmal vorsichtig so formulieren: Ich wünschte mir etwas mehr Demut im Handeln, etwas mehr Zweifel bei allem Handlungsdruck, etwas mehr Nachdenklichkeit bei aller Hektik, etwas mehr Vorsicht bei der Bildung von Selbstbestätigungskartellen. Kri- 
sen stellen infrage. Krisen, die uns unsere eigenen Handlungsgrenzen bis hin zu Ohnmachtserfahrungen aufzeigen, tun dies erst recht.

Krisen sind immer auch Neubesinnungszeiten. Von Dietrich Bonhoeffer habe ich den Lieblingssatz übernommen, dass es in besonderen Zeiten eigentlich nur zwei Dinge zu tun gibt: Beten und Tun des Gerechten. Erweitert man Beten hier einmal zu Kontemplation oder Meditation, so scheint mir die Mischung ein guter Schutz zu sein gleichermaßen gegen selbstverliebten Aktionismus und tatenloses Nachdenken. Der Impuls zum Handeln erwächst aus dem Unterstützungsbedarf des Anderen, nicht aus der Selbstkonstitution.

\section{Literaturverzeichnis}

Birnbaum, R./Ismar, G (2020): Bundestagspräsident zur Corona-Krise: Schäuble will dem Schutz des Lebens nicht alles unterordnen. Link: https:/www.tagesspiegel.de/politik/bunde stagspraesident-zur-corona-krise-schaeuble-will-dem-schutz-des-lebens-nicht-alles-unterord nen/25770466.html (zuletzt abgerufen am 13.06.2020).

Jusatz, H. J. (1938): Über das rhythmische Auftreten von Grippeepidemien und die Möglichkeit einer epidemiologischen Prognose, in: Zeitschrift für Hygiene und Infektionskrankheiten, H. 121, S. 185-207.

Lee, F. (2020): Corona verändert die Weltwirtschaft. Grenzen der Globalisierung. Link: https:// taz.de/Corona-veraendert-die-Weltwirtschaft/!5681077 (zuletzt abgerufen am 13.06.2020).

ORF (2020): Notstandsgesetze beschlossen. Ungarns Parlament faktisch entmachtet. Link: https://orf.at/stories/3159914/ (zuletzt abgerufen am 13.06.2020).

von Weizsäcker, C. F. (1991): Der Mensch in seiner Geschichte, München: Carl Hanser.

Walser, M. (2012): Nichts ist ohne sein Gegenteil wahr: Martin Walser über sich und sein Werk, Edition Quartino (P): SWR. 